\title{
Edge-to-edge repair: will it still be mainstream repair therapy in 2030?
}

\author{
Julia Stehli ${ }^{1}$, Gorav Ailawadi ${ }^{2}$, D. Scott Lim $^{1}$ \\ ${ }^{1}$ Advanced Cardiac Valve Center, University of Virginia, Charlottesville, VA, USA; ${ }^{2}$ Department of Cardiac Surgery, University of Virginia, \\ Charlottesville, VA, USA \\ Correspondence to: D. Scott Lim. Advanced Cardiac Valve Center, University of Virginia, 1215 Lee St., Charlottesville, VA 22908 , USA. \\ Email: SL9PC@hscmail.mcc.virginia.edu.
}

Submitted May 07, 2020. Accepted for publication Jun 12, 2020.

doi: 10.21037/acs-2020-mv-20

View this article at: http://dx.doi.org/10.21037/acs-2020-mv-20

\section{Introduction}

We are only just beginning to address the large number of patients with significant mitral regurgitation (MR), despite guidelines recommending operative repair in our asymptomatic patients with primary $M R$, and the limitations of medical therapy for secondary MR. Using history as a guide, less invasive therapies, including nonsurgical approaches, will be rapidly adopted once there is some evidence of similar mortality and MR reduction compared to gold standard open surgery.

\section{The past \& present}

In order to predict the future, one needs to understand the past and the present. Edge-to-edge therapy with MitraClip (Abbott Vascular, Santa Clara, CA, USA) was first designed to be a less invasive approach to repair MR, as initially envisioned by St Goar and colleagues (1). The MitraClip device was delivered via a transvenous, trans-septal approach and used a mechanical clip to oppose the mitral leaflets in the area of regurgitation.

\section{Degenerative MR}

In the first ever randomized clinical trial studying MR, the EVEREST II trial demonstrated a significant benefit in terms of safety with MitraClip over open surgical repair, although the outcomes in terms of MR reduction were not equivalent (2). At 5 years follow-up, $97 \%$ of surgical patients remained free from grade 3 or $4+$ MR versus $81 \%$ for MitraClip-treated patients. Interestingly, no differences in symptoms nor mortality were identified between MitraClip and surgical treatment strategies.

Based on results from a subset of patients in EVEREST II, MitraClip ultimately gained regulatory approval in 2011, with subsequent guideline recommendations for prohibitive surgical risk patients in primary or degenerative MR (3). One criticism of the process was that results from the most experienced mitral valve surgeons were compared with that of MitraClip operators, who were new to the procedure. With greater operator experience, along with improvements in imaging and technological refinement of the MitraClip device, the recent EXPAND study investigated the results of more than 1,000 consecutively enrolled subjects (4). For those with primary MR, treatment by MitraClip resulted in freedom from grade $3-4+M R$ in $96 \%$ of patients at 12 -month follow-up, as adjudicated by an echocardiographic core laboratory.

Coincident with this, another transcatheter edge-toedge repair device, the Pascal device (Edwards Lifesciences, Irvine, CA, USA), was more recently developed and studies regarding the device were initiated (CLASP study, NCT03706833). The Pascal device also has some potential design advantages over MitraClip, including wider coaptation surfaces, fewer frictional elements to reduce chordal entanglement risks and the use of a spacer element to theoretically optimize MR reduction. Early investigations of the Pascal device in the CLASP study demonstrated a similarly low complication rate and high success rate, with $98 \%$ freedom from grade 3-4+ MR at 30-day follow-up (5). If the newer MitraClip NT/XT and the Pascal device can demonstrate surgical-like, long-term durability for primary 
MR reduction, these procedures will become mainstream therapy.

\section{Functional MR}

In secondary (i.e., functional) MR, the primary therapeutic modality is heart failure medications with cardiac resynchronization therapy in appropriate patients. Surgical intervention has been comparatively limited in its adoption, due to scarce supporting data for improved mortality or heart failure hospitalization benefit and a high rate of late recurrent MR (6). For patients who, despite optimal guideline-directed medical therapy, remain symptomatic with severe functional MR, transcatheter mitral valve repair has recently gained the spotlight. The COAPT trial (i.e., cardiovascular outcomes assessment of the MitraClip percutaneous therapy for heart failure patients with functional mitral regurgitation) demonstrated that patients undergoing repair with MitraClip had significantly improved survival and symptoms, with lower hospitalization rates than the medical therapy arm (7). Remarkably, 95\% of patients undergoing MitraClip intervention in the COAPT trial had reductions in MR to lower than grade 3-4+. This in turn has and will continue to cement the role of MitraClip in the treatment of secondary MR in a population that otherwise has a $50 \%$ mortality and $90 \%$ hospitalization rate over five years (8). Consequentially, the Food and Drug Administration (FDA) approved the use of MitraClip for severe, symptomatic secondary MR refractory to medical treatment last year. Patient selection remains an important component of this therapy in those with secondary MR, as illustrated in the Mitra-FR trial (i.e., percutaneous repair with the MitraClip device for severe functional/secondary mitral regurgitation) (9). The Mitra-FR trial demonstrated a lack of therapeutic benefit in patients with larger left ventricular volumes and lesser degrees of MR. This led to the notion that only patients with MR 'disproportionate' to left ventricular dysfunction and size may benefit from the procedure (10).

Although demonstrating gradually improving and nearsurgical results without the associated risks and morbidity, percutaneous edge-to-edge repair has its limitations, such as dealing with underlying mitral stenosis pathology and the concern for preservation of future options, including transcatheter mitral valve replacement. There are currently no other mitral valve devices that have the proven safety and results equal to those of percutaneous edge-to-edge repair, nor with the depth of experience of over 100,000 patients treated worldwide.

\section{Conclusions}

Owing to the excellent safety profile, less invasive nature, improving results in MR reduction and mortality benefit, percutaneous edge-to-edge repair will likely still be mainstream therapy in 2030 for patients with degenerative MR at high surgical risk and for patients with functional MR despite medical therapy. Pending the results of trials in lower risk strata of degenerative MR, we anticipate its consideration for a broader range of patients with primary MR. Mitral pathology is often too complex and variable to be treated with a single tool alone. Therefore, percutaneous edge-to-edge repair will likely be one part of the transcatheter toolbox that also includes chordal replacement, annuloplasty and mitral valve replacement. As experience grows with these novel tools, percutaneous edgeto-edge repair will act as the gold standard. Ultimately, many tools will be necessary, but edge-to-edge therapies which continue to evolve and improve in order to remain the primary treatment for many patients with MR.

\section{Acknowledgments}

Funding: None.

\section{Footnote}

Conflicts of Interest: The authors have no conflicts of interest to declare.

Open Access Statement: This is an Open Access article distributed in accordance with the Creative Commons Attribution-NonCommercial-NoDerivs 4.0 International License (CC BY-NC-ND 4.0), which permits the noncommercial replication and distribution of the article with the strict proviso that no changes or edits are made and the original work is properly cited (including links to both the formal publication through the relevant DOI and the license). See: https://creativecommons.org/licenses/by-nc-nd/4.0/.

\section{References}

1. St Goar FG, Fann JI, Komtebedde J, et al. Endovascular edge-to-edge mitral valve repair: short-term results in a 
porcine model. Circulation 2003;108:1990-3.

2. Feldman T, Kar S, Elmariah S, et al. Randomized comparison of percutaneous repair and surgery for mitral regurgitation: 5-year results of EVEREST II. J Am Coll Cardiol 2015;66:2844-54.

3. Lim DS, Reynolds MR, Feldman T, et al. Improved functional status and quality of life in prohibitive surgical risk patients with degenerative mitral regurgitation after transcatheter mitral valve repair. J Am Coll Cardiol 2014;64:182-92.

4. Lim DS. Contemporary outcomes with MitraClip (NTR/ XTR) system in primary mitral regurgitation: results from the Global EXPAND Study. In: Proceedings from the 2020 Scientific Sessions of the American College of Cardiology, 2020.

5. Lim DS, Kar S, Spargias K, et al. Transcatheter valve repair for patients with mitral regurgitation: 30-day results of the CLASP study. JACC Cardiovasc Interv

Cite this article as: Stehli J, Ailawadi G, Lim DS. Edge-toedge repair: will it still be mainstream repair therapy in 2030? Ann Cardiothorac Surg 2021;10(1):158-160. doi: 10.21037/acs2020-mv-20
2019;12:1369-78.

6. Michler RE, Smith PK, Parides MK, et al. Two-year outcomes of surgical treatment of moderate ischemic mitral regurgitation. N Engl J Med 2016;374:1932-41.

7. Stone GW, Lindenfeld J, Abraham WT, et al. Transcatheter mitral-valve repair in patients with heart failure. N Engl J Med 2018;379:2307-18.

8. Goliasch G, Bartko PE, Pavo N, et al. Refining the prognostic impact of functional mitral regurgitation in chronic heart failure. Eur Heart J 2018;39:39-46.

9. Obadia JF, Messika-Zeitoun D, Leurent G, et al. Percutaneous repair or medical treatment for secondary mitral regurgitation. N Engl J Med 2018;379:2297-306.

10. Grayburn PA, Sannino A, Packer M. Proportionate and disproportionate functional mitral regurgitation: a new conceptual framework that reconciles the results of the MITRA-FR and COAPT trials. JACC Cardiovasc Imaging 2019;12:353-62. 